\title{
THE INTERVENTION OF COMPETITIVE AEROBIC GYMNASTICS ON BODY POSTURE
}

\section{Mariana CORDUN ${ }^{1}$, Mircea BRATU ${ }^{1}$, Cozeta-Anca MINCULESCU ${ }^{1}$, Oana STOIAN ${ }^{1}$, Alexandra GHEZEA ${ }^{1}$, Radu-Valentin CHIȚA ${ }^{1}$}

${ }^{1}$ National University of Physical Education and Sport, Faculty of Physical Therapy, Bucharest, Romania

*Corresponding author: mircea.bratu@yahoo.com

https://doi.org/10.35189/dpeskj.2021.60.4.6

\begin{abstract}
Practised as both a leisure activity and a mass and competitive sport, aerobic gymnastics is one of the most popular branches of gymnastics. As a mass and competitive sport, aerobic gymnastics has a complex motor repertoire that involves performing spectacular movements to music. The concept that has led to the emergence and development of competitive aerobic gymnastics is that of "spectator sport". The physical and mental demands and rigors of a competitive sport on those who want to be at the top of national and international rankings sometimes disturb their health balance. Purpose of the study: This study aims to determine whether the practice of competitive aerobics has any influence on the development and progression of scoliosis in female athletes who train for approximately 3 hours a day. Assessment methods: The assessment methods used to identify scoliosis are somatoscopy and various anthropometric measurements characteristic of three-dimensional postural deformities. Conclusion: Competitive sport can be considered an aggravating factor in the case of physical impairments due to the many positions and movements that an athlete has to perform, and the main causes of their occurrence are the excessive overloads imposed by competitive sport on the genetic background of each pubertal individual.
\end{abstract}

Keywords: competitive sport, aerobic gymnastics, physical impairments, scoliosis, somatoscopy.

\section{Introduction}

As a competitive sports discipline, aerobic gymnastics originated between the 1970s and 1980s, a period that marked one of the most significant "explosions" in the field of fitness.

The official request to introduce aerobic gymnastics into the competition programme of the International Gymnastics Federation (FIG) was made to the FIG Executive Committee on 30 October 1993, and shortly thereafter (1994), the FIG Congress in Geneva established the Aerobic Gymnastics Commission.

On 14 March 1995 (in Switzerland), the first course dedicated to aerobic gymnastics coaches and referees was organized, and at the end of that year (in December), the first edition of the World Aerobic Gymnastics Championships was held in Paris.

In 1996, aerobic gymnastics became one of the official sports disciplines of the FIG, and it has continued to develop as a sport ever since.

In Romania, aerobic gymnastics is one of the gymnastics disciplines for which the Romanian Gymnastics Federation is responsible, being one of the branches of Romanian sport with the most international results. Romania's first notable results in this sport were the silver medal in the trio event and the bronze medal in the team classification at the 1995 World Aerobic Gymnastics Championships (Paris). 
Aerobic gymnastics was born and developed around the concept of "show sport", and today this idea is more evident than ever in the performance of gymnasts during competitions.

One definition of aerobic gymnastics that we propose is: a branch of gymnastics that involves performing an exercise to music and incorporates both elements of difficulty and choreographed sequences of aerobic steps combined with upper limb movements, transitions, partnerships and constructions with different directions of movement and changes in the level of execution.

Nowadays, the high level of aerobic gymnastics can easily be seen through the prism of exercises composed of elements of increased difficulty, combinations of elements, transitions, partnerships and original constructions executed with virtuosity and elegance.

In the past, the main characteristic of aerobic gymnastics exercises was the increased difficulty of the elements, especially if their value was decreasing.

Gradually, the "race of difficulties" was no longer the only concern of specialists in the field, as they began to turn their attention to increasing the quality of exercises, which led to limiting the number of difficult elements required by the competition regulations, i.e., to a better correlation between the special requirements of the level of training and the age of athletes practising competitive aerobics.

The current approach to competitive aerobics allows athletes to stay in the competition circuit for a longer period, as the focus has been redirected towards the accuracy of technical execution, novelty of combinations, expressiveness and artistic presentation of compositions in competition.

The most recent performances of athletes in large-scale competitions have brought to the fore the "show" character of this sport through the increased level of artistic-choreographicmusical quality and the high-performance criteria fulfilled with impeccable technique and execution.

Today, aerobic gymnastics has a complex and varied motor repertoire composed of: aerobic step sequences (Aerobic Movement Patterns - AMP), difficulty elements, acrobatic elements, transitions, connections, partnerships and constructions.

The term Aerobic Movement Patterns (AMP) refers to movement structures made up of combinations of basic steps and upper limb movements. Aerobic step sequences are the choreographed part of an aerobic gymnastics exercise that must be performed in perfect harmony with the chosen music. They should include as many variations as possible in the level of impact with the ground (steps and jumps), direction of movement, dynamism, rhythm, intensity and continuity.

The basic steps specific to aerobic gymnastics are: March, Jog, Skip, Knee lift, Kick, Jack, Lunge.

The groups of elements that make up the technical content of aerobic gymnastics are: GROUP A - Dynamic Strength Elements, GROUP B - Static Strength Elements, GROUP C Jumps and Leaps Elements, GROUP D - Balance and Flexibility Elements.

In recent years, more acrobatic elements specific to artistic gymnastics have been included in the repertoire of aerobic gymnastics.

Chayun et al. (2020) mention that the competition exercises of great performers in aerobic gymnastics have various acrobatic elements in their structure. 
To be complete, an aerobic gymnastics exercise must have both aerobic step sequences and elements of difficulty, as well as transitions, connections, partnerships (physical interactions/ collaborations) and constructions (in the case of multimember events - couple, trio, group).

We consider that, in aerobic gymnastics, from the point of view of execution, the gymnastic act is performed in an aesthetic-artistic manner that acquires the character of a dance and is manifested by: elegance, plasticity, flow, unity, motor expressiveness, (varied) dynamism, correct segmental placement, safety and precision in conditions of multisegmental coordination, variety of rhythm and musical tempo, coordination between body movements and the particularities of the musical piece.

Gymnastic disciplines (artistic gymnastics, rhythmic gymnastics, aerobic gymnastics) are based on elements taken from the content of classical academic dance, a form of dance that requires a codified unitary technique and a specific methodology that aims to educate the dancer's abilities so that they can rise in space through the amplitude of their movements.

The specific means of classical academic dance included in the training of aerobic gymnasts are: jumps, balance elements, turns, pirouettes, various linking elements, movement variations as well as basic exercises necessary to prepare dancers to perfect the technique of body elements and artistic expression.

In terms of structure, an aerobic gymnastics exercise is based on three criteria: difficulty, execution and artistry. Each criterion contributes equally to the final score obtained by athletes in an aerobic gymnastics competition.

For the most accurate execution of technical elements specific to this sport, Bota and Urzeală (2013) recommend including exercises specific to the development of motor control in traditional training.

Mezei and Cristea (2014) state that the artistic skills of children and juniors need to be trained as early as possible for receiving higher scores on the artistic criterion.

Knowing in detail what the practice of aerobic gymnastics entails, Mezei and Bota (2016) state that an aerobic gymnastics exercise is composed within about two weeks, after which the process continues so that the choreographic composition is improved by adding elements of originality and reaching the accuracy of movements. The two authors mentioned above conclude that combining the fitness factor with consistent artistic training helps to maximise the choreographic concept, which is directly reflected in the athlete's performance.

According to Bota and Lăutaru (2016), by combining dance types (theatrical), genres (ballet, modern, contemporary) and styles (early, modern, post-modern), specialists working in the sphere of artistic sports can improve, innovate or refresh their knowledge of the human body, strategic vision and means of expression through which they can not only help their athletes reach the world elite but also create memorable compositions that open new horizons in aerobic gymnastics.

The uniqueness and beauty of the sport lies in the complexity and variety of movements performed by athletes in the competition exercise. As a competitive discipline, aerobic gymnastics is considered by outsiders (spectators) to be a sport that can affect health. Gymnastics is often thought to stunt growth, deform the spine or cause gymnasts to overdevelop their muscle mass. In reality, all these negative health effects are the consequences of poorly planned and applied training. 
We believe that the onset of spinal deformities such as scoliosis may occur relatively quickly in the context of competitive gymnastics if training does not aim to achieve a balanced volume of movements performed on both sides of the body as well as the optimal development of all muscle groups of the body, especially the paravertebral and abdominal muscles.

Scoliosis can be a good reason for a gymnast to give up competitive sport because this type of physical impairment can cause major changes in the spine.

Posture is defined as an attitude assumed by the body through an orthostatic, antigravity position that, through neuro-myo-arthrokinetic activities, maintains and transforms postural stability into the correct, normal posture or attitude, which is called orthostatic alignment position (Cordun, 1999).

One of the main passive elements of body posture is the spine, which is the supporting axis of the upper half of the human body.

The basic functions of the spine are:

- stability of the human body;

- protection of the spinal cord and spinal nerve roots;

- coordination and movement of the torso.

The vertebral column, consisting of several mobile bones (called vertebrae) that are surrounded by soft tissues, is an ideal structure that simultaneously fulfils the functions listed above and is therefore an optimised compromise of evolution (Putz \& Müller-Gerbl, 1996).

The vertebral column is made up of 24 mobile vertebrae whose order from top to bottom is: 7 cervical $(\mathrm{C}), 12$ thoracic $(\mathrm{T}), 5$ lumbar $(\mathrm{L}), 5$ united sacral $(\mathrm{S})$ vertebrae forming the sacrum and 3 to 5 united vertebrae forming the coccyx (Co).

The vertebral column is straight in the frontal plane and has the following alternating physiological curves in the sagittal plane, which offset each other. Thus, starting from the upper plane, the following curves are identified:

- cervical lordosis - determined by the position of the head; the anteriorly oriented convexity has a maximum curvature at the level of the $\mathrm{C} 7$ vertebra, which consists of 7 cervical vertebrae;

- dorsal kyphosis - determined by the positions of the head and scapular girdle; the posteriorly oriented convexity has a maximum curvature at the level of the D6 or D7 vertebrae, which consists of 12 thoracic vertebrae;

- lumbar lordosis - determined by the positions of the pelvis and abdomen; the anteriorly oriented convexity has a maximum curvature at the level of the L4 or L5 vertebrae, which consists of 5 lumbar vertebrae;

- sacrococcygeal kyphosis - defined by a fixed short curvature with a posteriorly oriented convexity.

The spine is part of the musculoskeletal core of the human body together with the hips, pelvis, proximal lower limbs and abdominal structures (Kibler et al., 2006).

The central musculoskeletal core fulfils the functions of stabilisation and force generation but is also involved in all activities of the extremities.

Scoliosis is a structural disorder involving an abnormal deformity of the spine in three planes: frontal, transverse and sagittal, and the resulting curves are called scoliotic curves. 
This deformity develops from a straight spine into a curved spine (characterised by a lateral deviation) and is followed by a deformity of the ribcage through an axial rotation of the spine.

Although scoliosis is a three-dimensional (3D) deformity of the spine, in reality, its diagnosis and treatment are based on radiographs from the front/back and the side, and thus the spinal deformity is assessed only in the frontal and sagittal (2D) planes, without including the axial plane (Illés et al., 2019). Given the above, in the clinical assessment of scoliosis based on radiographs, mathematical approaches have been developed to assess the amount of vertebral rotation in the axial plane.

In the SOSORT Guidelines (Negrini et al., 2018), the Scoliosis Research Society (SRS) recommends recognition of the diagnosis of scoliosis when the Cobb angle is equal to or greater than $10^{\circ}$ and there is definite rotation measured at the level of apical vertebra in the axial plane.

According to the American Association of Neurological Surgeons (2021), scoliosis is classified by aetiology as follows:

○ congenital scoliosis - occurs during the foetal development due to the malformation of spinal vertebrae and is present at birth;

○ neuromuscular scoliosis - develops secondary to a disorder of the neurological or muscular system and progresses faster than the other types of scoliosis.

o idiopathic scoliosis - its etiopathogenesis remains unknown despite basic scientific clinical and epidemiological research.

In the above classification, it is noted that, due to the diagnostic conditions of congenital and neuromuscular scoliosis, idiopathic scoliosis has an exclusion diagnosis, being certified only when the medical history, clinical evaluation and radiographs do not provide clear evidence of aetiology.

The classification of idiopathic scoliosis according to the age of onset is:

o infantile idiopathic scoliosis (IIS) - age group 0-3 years;

○ juvenile idiopathic scoliosis (JIS) - age group 4-10 years;

○ adolescent idiopathic scoliosis (AIS) - age group 10-18 years.

Of this classification, AIS is the most common paediatric spinal deformity that has the fastest progression during pubertal growth (Cheng et al., 2015).

During pubertal growth, the loss of mechanical stability of the spine results in deformity of the ribs and vertebral body, which is directly related to the unpredictability and severity of the scoliosis curve. Since the etiopathogenesis of AIS remains unknown, it should be diagnosed as early as possible because the scoliosis curve, especially at puberty, increases aggressively.

The risk of progression of the scoliotic curve in AIS is based on the following factors (Weinstein et al., 2008):

○ patient maturity: assessed by age at diagnosis, menarche status, remaining skeletal growth;

○ size of the scoliotic curve;

○ position of the peak of the scoliotic curve.

Over the years, studies on AIS have observed an increased percentage of cases of scoliosis among women. 
In the case of AIS, puberty is the possible period for the onset of idiopathic scoliosis, which is associated with an increased length of the vertical spine and a rapid increase in the scoliotic curvature. According to Rinsky and Gamble (1988), "the standing torso length of girls with scoliosis is slightly longer than that of controls, suggesting that the increased growth also places them at increased risk" (p. 184).

This increase in torse length in girls with AIS commonly occurs one year earlier than in girls without scoliosis. At this time of puberty, when the anterior spine is relatively too long compared to posterior bony elements, the continuous progression of the scoliosis curve reaches a critical point at which gravity and mechanical factors act autonomously, definitely causing lordosis with a convex vertebral rotation in the transverse plane due to anteroposterior length imbalance.

One of the causes of scoliosis would be central nervous system dysfunction together with impaired balance function (Barrack et al., 1984; Ford et al., 1984), which leads to the imbalance of load distribution in the vertebral body (Zetterberg et al., 1984).

Stable balance in the complexity of the phenomenon involves, through the central nervous system, proprioceptive integration of sensory systems (visual and vestibular ones, for example) to create a general representation of body position and movement. Possible failures of this stable balance may cause AIS to occur.

Aerobic gymnastics is a sport practised by graceful, rhythmic, slender and elegant female athletes whose height and weight are lower compared to girls of the same age.

The selection for aerobic gymnastics takes place before adolescence, around the age of 6 , and the main criteria are family history, body thinness, flexibility and agility.

For the development of motor skills, practical and theoretical training in aerobic gymnastics mainly develops the flexibility, coordination and balance of the athlete.

In aerobic gymnastics, flexibility, one of the quality factors influencing the range of motion, is expressed by joint mobility and the level of muscle stretch and elasticity. Regular exercise in daily training develops high levels of flexibility in the hip, spine and scapulohumeral rhythm, a prerequisite for the execution of all basic elements, i.e., jump, balance and rotation. This development of flexibility in aerobic gymnastics leads to a $100 \%$ prevalence of generalised joint laxity in athletes.

The characteristic of aerobic gymnastics athletes is their flat back posture, which is maintained during daily activities such as standing or walking. The flat back posture of aerobic gymnasts shows their tendency towards thoracic hypokinesis and lumbar hypolordosis.

The pace of their daily training, namely almost 3 hours per day, results in significant physical and mental overload for adolescent female athletes who start active aerobic gymnastics training from the age of 5. The factors considered to be of major importance in the aetiology of idiopathic scoliosis are: "nutrition, hormonal influence, genetic tendency, and spinal mechanics" (Harrington, 1977, p. 17). As can be seen, the factors in this list that have a direct connection with female athletes practising aerobic gymnastics are the following:

○ nutrition - the importance of athletes' weight for achieving maximum performance in this sport is well known, which is why they are $10 \mathrm{~kg}$ below the weight standards for their age; 
o hormonal influence - delayed growth and maturation, physical training from an early age and forced diet to maintain competitive weight cause delayed menarche and exposes for a longer period the growth plates to the influence of mechanical factors.

$\circ$ spinal mechanics - the influence of flexibility development and flat back posture in aerobic gymnastics was discussed above;

At the same time, so far there is no classification by aetiology of spinal deformities and therefore scoliosis for aerobic gymnasts.

In conclusion, there are many unanswered questions about AIS, but the most important controversy is the lack of information about its etiopathogenesis. Thus, not all factors leading to the onset, progression and development of the spinal deformity called AIS are known.

\section{Purpose of the study}

The study aims to determine whether the practice of competitive aerobics has any influence on the development and progression of scoliosis in female athletes who train for approximately 3 hours a day.

\section{Hypothesis}

The asymmetry of the spine in competitive aerobic gymnasts can determine significant differences in anteroposterior diameter and lateral tilt of the body.

\section{Methodology}

\section{Participants}

The research participants are members of the aerobic gymnastics team of the CS UNEFS Bucharest Club. They are aged between 10 and 18 years and meet the required criteria.

o Inclusion criteria: competitive aerobic gymnasts with at least 3 years of experience, athletes included in the national and international competition circuit, regular participation in training programmes (minimum 5 training sessions per week lasting approximately 3 hours).

- Exclusion criteria: age under 10 years, age over 18 years, athletes with less than 2 years of experience in competitive aerobics, athletes participating in mass sports festivals, frequent absence from training programmes, participation in a similar study.

The data collection period was April 2021, when athletes were in pre-competitive training.

\section{Methods}

The assessment methods used to identify scoliosis are somatoscopy and various anthropometric measurements characteristic of three-dimensional postural deformities. 


\section{Procedure}

Anthropometric measurements are represented by:

- Tomayer index - in the orthostatic position, the person is asked to perform flexion of the trunk on the pelvis; normally, medius-ground contact should be achieved; if the medius is maintained above the ground, the distance from the medius to the ground is measured with the tape measure, noting the negative value; if the palms are placed on the ground, then the person will stand on a raised surface, and the plant-medius distance will be measured with the tape measure, noting the positive value.

- lateral tilt - in the orthostatic position, the person is asked to tilt the trunk laterally, measuring the distance from the medius to the ground with a tape measure.

- chest perimeter - in the orthostatic position, the person is asked to perform abduction of the upper limbs; the tape measure is placed anteriorly at the xiphoid appendix and posteriorly at the inferior angle of the scapulae; the values obtained at rest, during inspiration and deep expiration are noted.

- anteroposterior diameter - in the orthostatic position, the person is asked to perform arm abduction and bend the elbows so that the palms are maintained at the occiput; the distance between the xiphoid appendix and the corresponding spinous process is measured with the anthropometric compass.

- rib hump assessment - in the orthostatic position, the person is asked to bend the trunk on the thighs; the therapist observes and notes the elevation of the hemithorax.

As for somatoscopy, the biacromial line, biscapular line and biceps line were inspected. The collected data were analysed with the Mann-Whitney $U$ test and Excel and presented using graphs.

\section{Results}

Figures 1, 2, 3 and 4 highlight the data collected following anthropometric measurements.

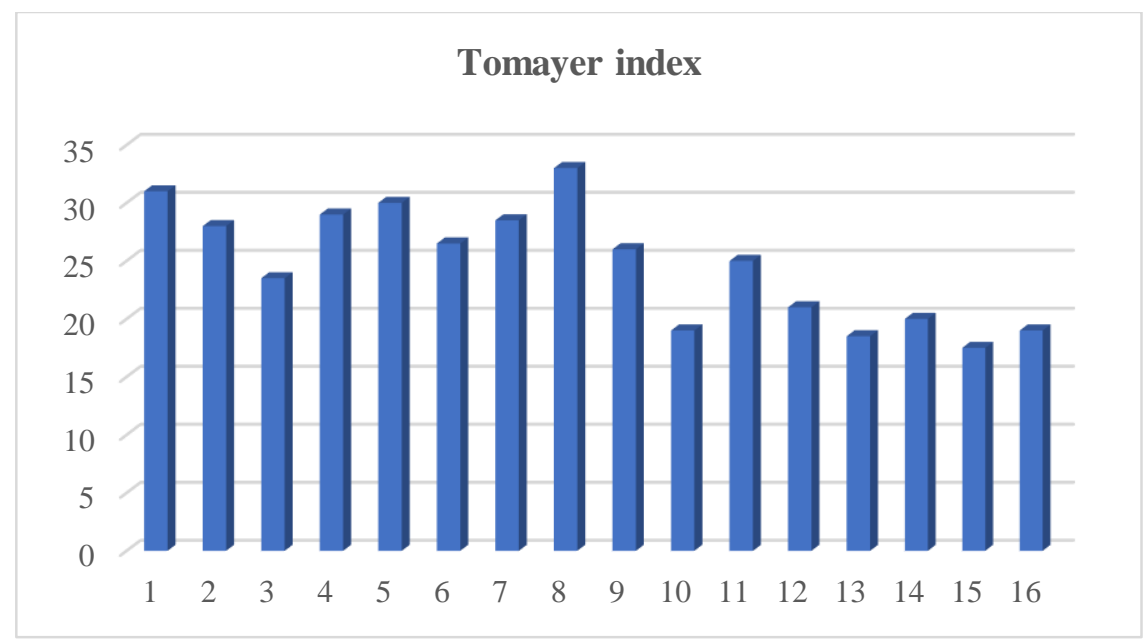

Figure 1. Tomayer index results 
All research participants have positive values on the Tomayer index measure. Athlete 8 has the highest value, namely $33 \mathrm{~cm}$. The lowest value is $17.5 \mathrm{~cm}$ and is observed in athlete 15. This aspect is justified by the different (reduced/increased) involvement in the training activity.

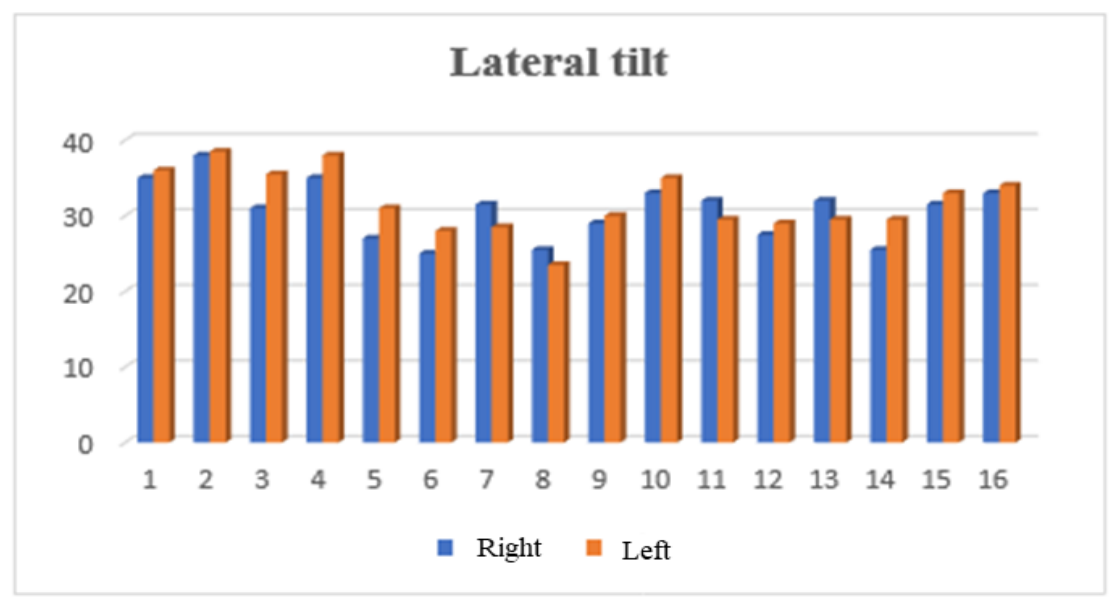

Figure 2. Lateral tilt results

Comparing the lateral tilt results on the right and left sides, no major differences are found between their values, which is justified by the balanced demands on the paravertebral muscles in the motor gestures specific to aerobic gymnastics.

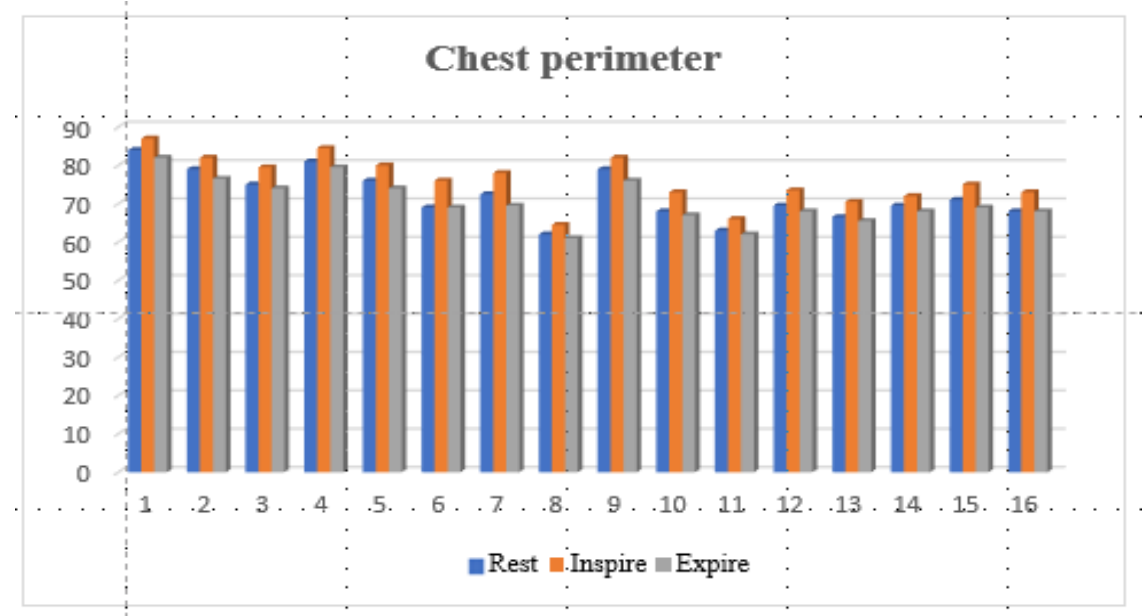

Figure 3. Chest perimeter results

Comparing the resting, inspiratory and expiratory measurements of the chest perimeter, the first athlete shows the highest values, followed by athlete 4, which is explained by individual somatometric and functional characteristics. 


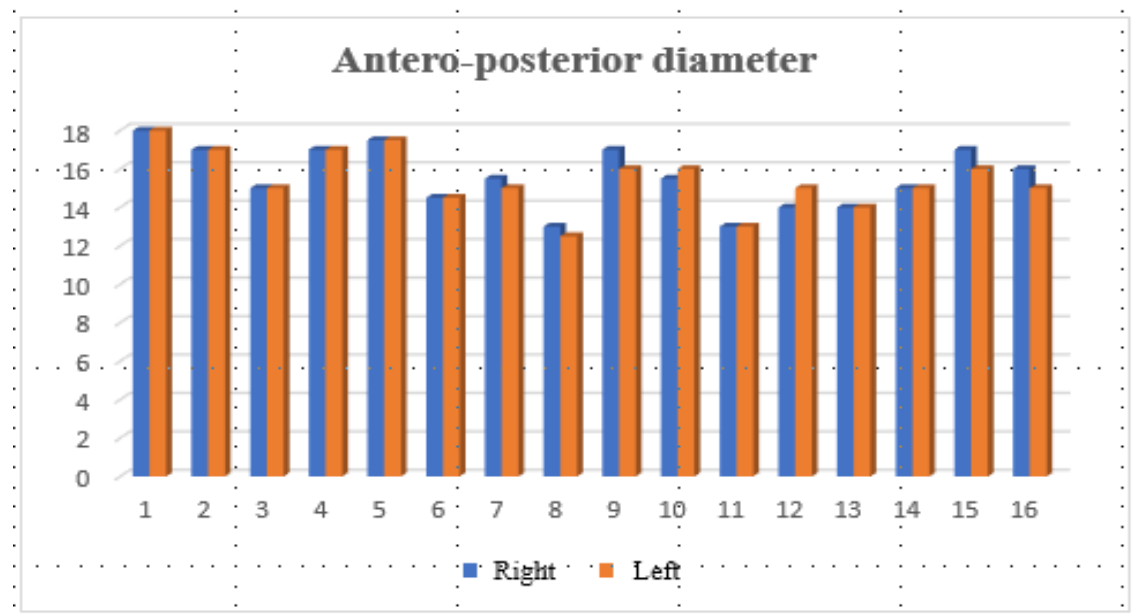

Figure 4. Anteroposterior diameter results

By measuring the anteroposterior diameter at the two hemithorax, it can be seen that the recorded values do not show significant differences, most of the tested athletes showing symmetry on both the right and left sides. This is justified by the relatively symmetrical demands characteristic of the motor gestures performed in aerobic gymnastics.

No significant differences in scoliosis were found in the 16 participants. Statistically, the detection of costal gibbosity cannot be quantified because no specific measuring instruments have been used. Table 1 shows the statistical data of the measurements.

Table 1. Collected data processing

\begin{tabular}{lcccccccc}
\hline \multirow{2}{*}{$\begin{array}{c}\text { Parameters } \\
\text { index }(\mathrm{cm})\end{array}$} & \multicolumn{2}{c}{$\begin{array}{c}\text { Lateral tilt } \\
(\mathrm{cm})\end{array}$} & \multicolumn{2}{c}{$\begin{array}{c}\text { Chest perimeter } \\
(\mathrm{cm})\end{array}$} & \multicolumn{2}{c}{$\begin{array}{c}\text { Anteroposterior } \\
\text { diameter }(\mathrm{cm})\end{array}$} \\
\cline { 3 - 9 } & & Right & Left & Rest & Inspire & Expire & Right & Left \\
\hline Minimum & 17.5 & 25 & 23.5 & 62 & 64.5 & 61 & 13 & 12.5 \\
Maximum & 33 & 38 & 38.5 & 84 & 87 & 82 & 18 & 18 \\
Average & 24.71 & 30.71 & 31.78 & 72.06 & 76.03 & 70.56 & 15.56 & 15.40625 \\
Median & 25.5 & 31.5 & 30.5 & 70.25 & 75.5 & 69 & 15.5 & 15 \\
Standard deviation & 5.01 & 3.82 & 4.10 & 6.39 & 6.33 & 5.93 & 1.59 & 1.52 \\
Coefficient of & $20.31 \%$ & $12.44 \%$ & $12.92 \%$ & $8.88 \%$ & $8.33 \%$ & $8.41 \%$ & $10.22 \%$ & $9.93 \%$ \\
variation & & & & & & & & \\
\hline
\end{tabular}

For the Tomayer index, the minimum value recorded is $17.5 \mathrm{~cm}$, and the maximum value is $33 \mathrm{~cm}$. Thus, it is observed that gymnasts have an accentuated mobility in accordance with the execution requirements for the technical elements of difficulty and the artistic, choreographic elements.

The gymnast with the smallest waist had the highest mobility/flexibility parameter.

For the lateral tilt to the right side, the average is $30.71 \mathrm{~cm}$, and to the left side, it is around $31.78 \mathrm{~cm}$. Thus, it results that the physical training was performed correctly with muscle toning on both hemi-bodies, according to the individual training plans.

The calculated median chest perimeter is $70.25 \mathrm{~cm}$ at rest, $75.5 \mathrm{~cm}$ at inspiration and 69 $\mathrm{cm}$ at expiration. It is noted that there are small differences between athletes, which reveals a good direction of sports training in accordance with the oxygen demands during exercise. 
The calculated standard deviation of the anteroposterior diameter is $1.59 \mathrm{~cm}$ for the right hemithorax and $1.52 \mathrm{~cm}$ for the left hemithorax.

Subjectivity may be present in the rib hump assessment, as the identification of an ascending hemithorax is influenced by the therapist's experience.

Table 2 shows the statistical data processed with the nonparametric Mann-Whitney U test, comparing the results recorded with the same anthropometric measurement.

Table 2. Mann-Whitney U test - Lateral tilt/ Chest perimeter/ Anteroposterior diameter

\begin{tabular}{lcc}
\hline Anthropometric measurement & \multicolumn{2}{c}{ Mann-Whitney U test } \\
\cline { 2 - 3 } & U-value & p-value \\
\hline Lateral tilt & 109.5 & 0.4965 \\
Chest perimeter (inspire-expire) & 70 & 0.30 \\
Anteroposterior diameter & 121 & 0.81034 \\
\hline
\end{tabular}

For the lateral tilt, the result is insignificant being less than 0.05 .

For the chest perimeter, the result is insignificant being less than 0.05.

For the anteroposterior diameter, the result is insignificant being less than 0.05 , and the $\mathrm{z}$ score is 0.24 .

\section{Discussion}

The particularities of the technical content of aerobic gymnastics require athletes to perform multiple variations of difficulty elements and aerobic step sequences. In order to obtain the best possible score in all components of the final score, the gymnast must demonstrate strength, speed, mobility, continuity, fluidity, plasticity, ambidexterity and expressiveness in movement.

Compared to other studies on similar sports such as ballet and dance, whose characteristics largely coincide with aerobic gymnastics, it is found that their practice contributes to an increased incidence of scoliosis.

Jandric (2015) found that the incidence rate of scoliosis in the two categories of investigated participants differed significantly: in ballet dancers, the incidence of scoliosis reached $30 \%$, while in non-athletes, it was only $3 \%$. The results of the study also showed that ballerinas had a 12.4 times higher risk of developing scoliosis than girls of the same age who did not practise ballet or dance. Compared to the non-athlete group (3\%), ballerinas $(70 \%)$ had higher values in terms of mobility (hypermobility), but no statistically significant association could be established between hypermobility, menarche, BMI or the number of hours of training (dance) performed per week.

We believe that, unlike ballet and dance where training is based on unilateral (asymmetrical) work and hypermobility, aerobic gymnastics additionally involves musclebuilding exercises that create the support for maintaining normal (correct) body posture.

In the study by Kenanidis et al. (2010), it has been concluded that the relationship between adolescent idiopathic scoliosis (AIS) and sport is rather vague. Therefore, in this case, sport is often seen as both a causative factor and a means of treatment, especially for adolescents who practise different sports. This idea also results from the data obtained in our study. 
Performing aerobic step sequences involves the engagement in movement of the lower and upper limbs both simultaneously and separately, in all anatomical planes and axes.

Most of the technical difficulty elements require to be performed on both the right and left sides of the body in order to be included later (in higher age categories - junior I, senior) in different combinations of elements that increase the value of the "Difficulty" component.

The plastic and aesthetic execution of the movements included in the competition exercise is based on well-developed motor control and harmonious muscles that reach higher indices of manifestation.

These characteristics of aerobic gymnastics have positive effects on the health of gymnasts, as they require conscious and consistent training carried out through movements executed in approximately equal proportions on both sides of the body.

Such sports training can be considered a form of maintaining correct body posture, which excludes the existence of scoliotic-type deformities of the spine.

\section{Conclusion}

Competitive sport can be considered an aggravating factor in the case of physical impairments due to the numerous positions and movements that an athlete has to perform, and the main causes of their occurrence are the excessive overloads imposed by competitive sport on the genetic background of each pubertal individual.

Statistical processing reveals that the hypothesis according to which "the asymmetry of the spine in competitive aerobic gymnasts can determine significant differences in anteroposterior diameter and lateral tilt of the body" is invalid, the analysed parameters highlighting that aerobic gymnastics is not the determining cause of scoliosis.

To successfully meet the performance criteria in aerobic gymnastics, athletes must have well-developed and balanced muscle tone that prevents physical impairments.

A training programme based on the practice of symmetrical body movements as much as possible prevents the development of spinal malposition.

The predominantly asymmetrical work of competitive athletes in conditions of excessive overload creates a context conducive to the onset of scoliosis with a negative impact on both their sports activity and quality of life.

\section{Authors' Contribution}

All authors had an equal contribution and accepted the final manuscript.

\section{References}

American Association of Neurological Surgeons. (2021). Scoliosis. https://www.aans.org/en/Patients/Neurosurgical-Conditions-and-Treatments/Scoliosis

Barrack, R. L., Whitecloud 3rd, T. S., Burke, S. W., Cook, S. D, \& Harding, A. F. (1984). Proprioception in idiopathic scoliosis. Spine, 9(7), 681-685.

https://doi.org/10.1097/00007632-198410000-00005 
Bota, A., \& Lăutaru, C. (2016). Contemporary trends in choreography - Potential for enhancing the artistic preparation in rhythmic and aerobic gymnastics. European Proceedings of Social and Behavioural Sciences, 11(4), 22-29.

http://dx.doi.org/10.15405/epsbs.2016.06.4

Bota, A., \& Urzeală, C. (2013). Correlative aspects regarding functional stress and neuromuscular control in high performance aerobic gymnastics - Individual events. Procedia - Social and Behavioral Sciences, 93, 2090-2094. https://doi.org/10.1016/J.SBSPRO.2013.10.171

Chayun, D., Kletsov, K., \& Manzheley, I. (2020). Acrobatics as a new trend in Aerobic Gymnastics. Journal of Physical Education and Sport, 20(2), 759-767. DOI: $10.7752 /$ jpes.2020.02109

Cheng, J. C., Castelein, R. M., Chu, W. C., Danielsson, A. J., Dobbs, M. B., Grivas, T. B., Gurnett, C. A., Luk, K. D., Moreau, A., Newton, P. O., Stokes, I. A., Weinstein, S. L., \& Burwell, R. G. (2015). Adolescent idiopathic scoliosis. Nature Reviews. Disease Primers, 1: 15030. https://doi.org/10.1038/nrdp.2015.30

Cordun, M. (1999). Postura corporală normală și patologică [Normal and pathological body posture]. ANEFS.

Ford, D. M., Bagnall, K. M., McFadden, K. D., Greenhill, B. J., \& Raso, V. J. (1984). Paraspinal muscle imbalance in adolescent idiopathic scoliosis. Spine, 9(4), 373-376. https://doi.org/10.1097/00007632-198405000-00008

Harrington, P. R. (1977). The etiology of idiopathic scoliosis. Clinical Orthopaedics and Related Research, 126, 17-25. PMID: 598109

Illés, T. S., Lavaste, F., \& Dubousset, J. F. (2019). The third dimension of scoliosis: The forgotten axial plane. Orthopaedics \& Traumatology: Surgery \& Research, 105(2), 351359. https://doi.org/10.1016/j.otsr.2018.10.021

Jandric, S. (2015). Scoliosis and sport. Sport Logia, 11(1), 1-10. https://doi.org/10.5550/sgia.151101.en.001j

Kenanidis, E. I., Potoupnis, M. E., Papavasiliou, K. A,, Sayeg, F. E,, \& Kapetanos, G. A. (2010). Adolescent idiopathic scoliosis in athletes: Is there a connection? The Physician and Sportsmedicine, 38(2), 165-170. https://doi.org/10.3810/psm.2010.06.1795

Kibler, W. B., Press, J., \& Sciascia, A. (2006). The role of core stability in athletic function. Sports Medicine, 36(3), 189-198. https://doi.org/10.2165/00007256-200636030-00001

Mezei, M., \& Bota, A. (2016). Conceptual and practical aspects in the preparation of a choreographic routine in high performance aerobic gymnastics - Example of best practice. Ovidius University Annals, Series Physical Education and Sport/Science, Movement and Health, XVI(2), 570-575.

Mezei, M., \& Cristea, O. (2014). Performance criteria in Aerobic Gymnastics - Impact on the sportive training. Procedia - Social and Behavioral Sciences, 117, 367-373. https://doi.org/10.1016/j.sbspro.2014.02.229

Negrini, S., Donzelli, S., Aulisa, A. G., Czaprowski, D., Schreiber, S., de Mauroy, J. C., Diers, H., Grivas, T. B., Knott, P., Kotwicki, T., Lebel, A., Marti, C., Maruyama, T., O’Brien, J., Price, N., Parent, E., Rigo, M., Romano, M., Stikeleather, L., ... Zaina, F. (2018). 2016 SOSORT guidelines: Orthopaedic and rehabilitation treatment of idiopathic scoliosis during growth. Scoliosis and Spinal Disorders, 13: 3. https://doi.org/10.1186/s13013-017-0145-8

Putz, R. L., \& Müller-Gerbl, M. (1996). The vertebral column - A phylogenetic failure? A theory explaining the function and vulnerability of the human spine. Clinical Anatomy, 9(3), 205-212. https://doi.org/10.1002/(sici)1098-2353(1996)9:3\%3C205::aid-ca12\%3E3.0.co;2-v 
Rinsky, L. A., \& Gamble, J. G. (1988). Adolescent idiopathic scoliosis. Western Journal of Medicine, 148(2), 182-191. PMID: 3279708

Shahin, F., Urak, Ö., \& Akkaya, N. (2019). Evaluation of balance in young adults with idiopathic scoliosis. Turkish Journal of Physical Medicine and Rehabilitation, 65(3), 236243. https://doi.org/10.5606/tftrd.2019.2825

Weinstein, S. L., Dolan, L. A., Cheng, J. C., Danielsson, A., \& Morcuende, J. A. (2008). Adolescent idiopathic scoliosis. Lancet, 371(9623), 1527-1537. https://doi.org/10.1016/S0140-6736(08)60658-3

Zetterberg, C., Björk, R., Örtengren, R., \& Andersson, G. B. J. (1984). Electromyography of the paravertebral muscles in idiopathic scoliosis: Measurements of amplitude and spectral changes under load. Acta Orthopaedica Scandinavica, 55(3), 304-309.

https://doi.org/10.3109/17453678408992362 